\title{
Stability and Bifurcation Analysis of a Nonlinear Discrete Logistic Model with Delay
}

\author{
Daiyong Wu, ${ }^{1}$ Hai Zhang, ${ }^{1,2}$ Jinde Cao, ${ }^{2,3}$ and Tasawar Hayat ${ }^{3,4}$ \\ ${ }^{1}$ Department of Mathematics, Anqing Normal University, Anqing, Anhui 246133, China \\ ${ }^{2}$ Department of Mathematics, Research Center for Complex Systems and Network Sciences, Southeast University, \\ Nanjing, Jiangsu 210096, China \\ ${ }^{3}$ Department of Mathematics, Faculty of Science, King Abdulaziz University, Jeddah 21589, Saudi Arabia \\ ${ }^{4}$ Department of Mathematics, Quaid-i-Azam University, Islamabad 44000, Pakistan
}

Correspondence should be addressed to Hai Zhang; zhanghai0121@163.com

Received 18 August 2013; Accepted 30 October 2013

Academic Editor: Zhan Zhou

Copyright (C) 2013 Daiyong Wu et al. This is an open access article distributed under the Creative Commons Attribution License, which permits unrestricted use, distribution, and reproduction in any medium, provided the original work is properly cited.

We consider a nonlinear discrete logistic model with delay. The characteristic equation of the linearized system at the positive equilibrium is a polynomial equation involving high order terms. We obtain the conditions ensuring the asymptotic stability of the positive equilibrium and the existence of Neimark-Sacker bifurcation, with respect to the parameter of the model. Based on the bifurcation theory, we discuss Neimark-Sacker bifurcation direction and the stability of bifurcated solutions. Finally, some numerical simulations are performed to illustrate the theoretical results.

\section{Introduction}

Logistic type models are often used to model a single species dynamics, for example, the underlying dynamics of tumour cells [1-4]. Moreover, time delay is sometimes necessary to better reflect the description of real processes $[5,6]$. The continuous single population model described by the ordinary differential equations has been studied very deeply [7-9]. There are also very many results concerning the discrete logistic model (see [10-15] and references therein).

In [13], Zhou and Zou considered a discrete logistic equation:

$$
x(n+1)=x(n) \exp \left[r(n)\left(1-\frac{x(n)}{K(n)}\right)\right],
$$

where $\{r(n)\}$ and $\{K(n)\}$ are positive $\omega$-periodic sequences. Sufficient conditions were obtained for the existence of a positive and globally asymptotically stable $\omega$-periodic solution.

In [14], Sun and Li considered the qualitative analysis of the following discrete logistic equation with several delays:

$$
x_{n+1}=x_{n} \exp \left(\sum_{i=1}^{m} r_{i}\left(1-\frac{x_{n-k_{i}}}{K}\right)\right),
$$

where $r_{i} \in(0, \infty)$ for $i=1,2, \ldots, m, k_{i}(i=1,2, \ldots, m)$ are positive integers, and $K \in(0, \infty)$. They obtained sufficient conditions for the global attractivity of all positive solutions about the positive equilibrium of model (2). Moreover, the oscillation about the positive equilibrium of model (2) was also discussed.

Chen and Zhou [15] discussed the following nonlinear periodic delay difference equation:

$$
\begin{array}{r}
x_{n+1}=x_{n} \exp \left[a_{n}+b_{n} x_{n-m \omega}^{p}-c_{n} x_{n-m \omega}^{q}\right], \\
n=0,1,2, \ldots,
\end{array}
$$

where $\left\{a_{n}\right\},\left\{b_{n}\right\}$, and $\left\{c_{n}\right\}$ are periodic sequences with a common period $\omega, a_{n}>0, c_{n}>0, m, \omega$ are positive integers, and $p, q$ are positive constants with $q>p$. Some sufficient conditions for the global attractivity and oscillation about the periodic solution of (3) were obtained.

Recently, Liu et al. [16] investigated the stability and bifurcation of a class of discrete-time Cohen-Grossberg neural networks with delays. Han and Liu [17] discussed the stability and bifurcation for a discrete-time model of Lotka-Volterra type with delay. He and Cao [18] studied the explicit formula for determining the direction of Neimark-Sacker bifurcation 
and the stability of periodic solution by using the normal form method and the center manifold theory.

To the best of our knowledge, no similar results have been reported regarding the nonlinear discrete logistic model with delay. In this paper, we are interested in the bifurcation analysis and the direction and the stability of the NeimarkSacker bifurcations for the following nonlinear discrete logistic model:

$$
\begin{array}{r}
x_{n+1}=x_{n} \exp [ \\
\left.n\left(1-a_{1} x_{n-m}-a_{2} x_{n-m}^{2}\right)\right], \\
n=0,1,2, \ldots ; \quad m \in \mathbb{Z}^{+},
\end{array}
$$

where $r, a_{1}$, and $a_{2}$ are positive constants, together with the initial condition

$$
x_{n}=\phi_{n}>0, \quad n \in\{-m,-m+1, \ldots, 0\} .
$$

Our works focus on the stability and bifurcation analysis and the direction analysis of the Neimark-Sacker bifurcations by applying the center manifold theorem and the normal form theory. The method of the paper is similar to the work of Yuri [19].

The paper is organized as follows. In Section 2, we analyze the distribution of the characteristic equation associated with the model (4) and obtain the existence of the Neimark-Sacker bifurcation. In Section 3, the direction and stability of closed invariant curve from the Neimark-Sacker bifurcation of the model (4) are determined. In Section 4, some numerical simulations are performed to illustrate the theoretical results. A brief discussion is given in Section 5.

\section{Stability Analysis}

In this section, we will employ the techniques of Guo et al. [20] to study the distribution of the characteristic roots of model (4). Then we will obtain the stability of the positive equilibrium and the existence of local Neimark-Sacker bifurcations. Clearly, model (4) has a unique positive equilibrium $x^{*}=2 /\left(\sqrt{a_{1}^{2}+4 a_{2}}+a_{1}\right)$.

Set $y_{n}=x_{n}-x^{*}$; then there follows that

$$
\begin{aligned}
y_{n+1}= & \left(y_{n}+x^{*}\right) \\
& \times \exp \left[r\left(1-a_{1}\left(y_{n-m}+x^{*}\right)-a_{2}\left(y_{n-m}+x^{*}\right)^{2}\right)\right]-x^{*} .
\end{aligned}
$$

By introducing a new variable $Y_{n}=\left(y_{n}, y_{n-1}, \ldots, y_{n-m}\right)^{T}$, we can rewrite (6) in the form

$$
Y_{n+1}=F\left(Y_{n}\right) \text {, }
$$

where $F=\left(F_{0}, F_{1}, \ldots, F_{m}\right)^{T}$ and

$$
F_{k}=\left\{\begin{aligned}
\left(y_{n}+x^{*}\right) & \\
\exp \left[r \left(1-a_{1}\left(y_{n-m}+x^{*}\right)\right.\right. & \\
\left.\left.-a_{2}\left(y_{n-m}+x^{*}\right)^{2}\right)\right]-x^{*}, & k=0, \\
y_{n-k+1}, & 1 \leq k \leq m .
\end{aligned}\right.
$$

Clearly, the origin is a fixed point of (7) and the linear part of (7) is

$$
Y_{n+1}=A Y_{n}
$$

where

$$
A=\left(\begin{array}{ccccc}
1 & 0 & \cdots & 0 & -r\left(a_{1}+2 a_{2} x^{*}\right) x^{*} \\
1 & 0 & \cdots & 0 & 0 \\
0 & 1 & \cdots & 0 & 0 \\
\vdots & \vdots & \ddots & \vdots & \vdots \\
0 & 0 & \cdots & 1 & 0
\end{array}\right)
$$

The characteristic equation of $A$ is given by

$$
P(\lambda)=\lambda^{m+1}-\lambda^{m}+r\left(a_{1}+2 a_{2} x^{*}\right) x^{*}=0 .
$$

Lemma 1. There exists $\bar{r}>0$ such that for $0<r<\bar{r}$ all roots of (11) have modulus less than one.

Proof. When $r=0$, (11) becomes

$$
\lambda^{m+1}-\lambda^{m}=0
$$

The equation has, at $r=0$, an $m$-fold $\operatorname{root} \lambda=0$ and a simple $\operatorname{root} \lambda=1$.

Consider the root $\lambda(r)$ such that $|\lambda(0)|=1$. This root depends continuously on $r$ and is a differential function of $r$. From (11), we have

$$
\begin{aligned}
\left.\frac{d|\lambda|^{2}}{d r}\right|_{r=0, \lambda=1} & =\left.\left[\lambda \frac{d \bar{\lambda}}{d r}+\bar{\lambda} \frac{d \lambda}{d r}\right]\right|_{r=0, \lambda=1} \\
& =-2\left(a_{1}+2 a_{2} x^{*}\right) x^{*}<0 .
\end{aligned}
$$

Consequently, $|\lambda|<1$ for all sufficient small $r>0$. Thus, all roots of (11) are inside the unit circle for sufficient small positive $r$, and the existence of the maximal $\bar{r}$ follows.

In the sequel, we define a parametric curve $\Sigma$ with

$$
\begin{aligned}
& u(t)=\cos (m+1) t-\cos m t, \\
& v(t)=\sin (m+1) t-\sin m t .
\end{aligned}
$$

Let $\theta(t)=v(t) / u(t)$. It is easy to see that $\theta^{\prime}(t)>0$ for all $t \in \mathbb{R}$ such that $u(t) \neq 0$. Therefore, as $t$ increases from 0 to $\pi$, the corresponding point $(u(t), v(t))$ on the curve $\Sigma$ moves from origin and anticlockwise around origin. Moreover, it follows from $u(t)^{2}+v(t)^{2}=2(1-\cos t)$ that the part of the curve $\Sigma$ with parameter $t \in[0, \pi]$ is simple; that is, it cannot intersect itself. Let $0 \leq \omega_{0} \leq \omega_{1}<\omega_{2}<\cdots<\omega_{m}<\omega_{m+1} \leq \pi$ be the $m+2$ zeros of $v(t)$ in the interval $[0, \pi]$ and $c_{j}=u\left(\omega_{j}\right)$ $(j \in\{0,1,2, \ldots, m+1\})$. Obviously, we have

$$
\begin{gathered}
\omega_{0}=0, \quad \omega_{j}=\frac{2 j-1}{2 m+1} \pi, \quad j=1,2, \ldots, m+1, \\
c_{j}=2(-1)^{j} \sin \frac{\omega_{j}}{2}, \quad j=0,1,2, \ldots, m+1 .
\end{gathered}
$$

Moreover, from the anticlockwise property of the curve $\Sigma$, we further have that

$$
(-1)^{j} v^{\prime}\left(\omega_{j}\right)>0, \quad j \in\{0,1,2, \ldots, m+1\} .
$$


From $u(t)^{2}+v(t)^{2}=2(1-\cos t)$, it follows that $0 \leq$ $\sqrt{u(t)^{2}+v(t)^{2}} \leq 2$. This means that the curve is contained in the region

$$
\left\{(u, v) \in \mathbb{R}^{2} \mid 0 \leq \sqrt{u(t)^{2}+v(t)^{2}} \leq 2\right\} .
$$

Accordingly, we set

$$
r_{k}=\frac{2}{\left(a_{1}+2 a_{2} x^{*}\right) x^{*}} \sin \frac{\omega_{2 k-1}}{2}, \quad k=1,2, \ldots,\left[\frac{m}{2}\right]+1,
$$

where $[\cdot]$ is the greatest integer function.

By Lemma 1 and the idea of Guo et al. (see Lemma 1 in [20]), we can obtain the distribution of the roots of the characteristic equation (11).

Lemma 2. Consider the following.

(i) $P(\lambda)$ has zeros on the unit circle if and only if $r=r_{k}$ for some $k \in\{1,2, \ldots,[m / 2]+1\}$. Moreover, if $r=r_{k}$ for some $k \in\{1,2, \ldots,[m / 2]+1\}$, then all the zeros of $P(\lambda)$ of modulus 1 are $e^{ \pm i \omega_{2 k-1}}$, which are simple.

(ii) $P(\lambda)$ has a simple zero -1 if and only if $m$ is even and $r=2 /\left(a_{1}+2 a_{2} x^{*}\right) x^{*}$.

(iii) For a fixed $k \in\{1,2, \ldots,[m / 2]+1\}$, there exist $\delta>0$ and a $C^{1}$-mapping $\lambda:\left(r_{k}-\delta, r_{k}+\delta\right) \rightarrow \mathbb{C}$ such that $\lambda\left(r_{k}\right)=e^{i \omega_{2 k-1}}$ and $\lambda(r)$ is a zero of $P(\lambda)$ for all $r \in\left(r_{k}-\delta, r_{k}+\delta\right)$.

(iv) All zeros of $P(\lambda)$ are inside the unit circle if and only if

$$
0<r<r_{1}=\frac{2}{\left(a_{1}+2 a_{2} x^{*}\right) x^{*}} \sin \frac{\pi}{2(2 m+1)} \text {. }
$$

Now we verify that the transversality condition is satisfied.

Lemma 3. Along (11), one obtains

$$
\left.\frac{d|\lambda|^{2}}{d r}\right|_{r=r_{k}}>0
$$

where $k=1,2, \ldots,[m / 2]+1$.

Proof. By Lemma 2(i), from (14) and (16), it follows that

$$
\begin{aligned}
\left.\frac{d|\lambda|^{2}}{d r}\right|_{r=r_{k}}= & {\left.\left[\lambda \frac{d \bar{\lambda}}{d r}+\bar{\lambda} \frac{d \lambda}{d r}\right]\right|_{r=r_{k}} } \\
= & -\left.2 \Re\left[\bar{\lambda} \frac{\left(a_{1}+2 a_{2} x^{*}\right) x^{*}}{(m+1) \lambda^{m}-m \lambda^{m-1}}\right]\right|_{r=r_{k}} \\
= & -2\left(a_{1}+2 a_{2} x^{*}\right) x^{*} \\
& \times \frac{(m+1) \cos (m+1) \omega_{2 k-1}-m \cos m \omega_{2 k-1}}{(m+1)^{2}+m^{2}-2 m(m+1) \cos \omega_{2 k-1}} \\
= & -2\left(a_{1}+2 a_{2} x^{*}\right) x^{*} \\
& \times \frac{v^{\prime}\left(\omega_{2 k-1}\right)}{(m+1)^{2}+m^{2}-2 m(m+1) \cos \omega_{2 k-1}}>0 .
\end{aligned}
$$

Remark 4. Since $\omega_{2 k-1} \neq \pi / 2,2 \pi / 3, \quad e^{i s \omega_{2 k-1}} \neq 1$ for $s=$ $1,2,3,4(k=1,2, \ldots,[(m-1) / 2]+1)$; that is, the nondegeneracy condition is satisfied.

Theorem 5. Consider the following.

(i) If

$$
0<r<r_{1}=\frac{2}{\left(a_{1}+2 a_{2} x^{*}\right) x^{*}} \sin \frac{\pi}{2(2 m+1)},
$$

then the fixed point of model (4) $x^{*}$ is asymptotically stable.

(ii) If

$$
r>r_{1}=\frac{2}{\left(a_{1}+2 a_{2} x^{*}\right) x^{*}} \sin \frac{\pi}{2(2 m+1)},
$$

then the fixed point of model (4) $x^{*}$ is unstable.

(iii) If

$$
\begin{aligned}
r & =r_{k} \\
& =\frac{2}{\left(a_{1}+2 a_{2} x^{*}\right) x^{*}} \sin \frac{\omega_{2 k-1}}{2}, \quad k=1,2, \ldots,\left[\frac{m-1}{2}\right]+1,
\end{aligned}
$$

then the model (4) undergoes a Neimark-Sacker bifurcation at the positive fixed point $x^{*}$; that is, a unique closed invariant curve bifurcates form $x^{*}$. Moreover, if $m$ is even, the model (4) undergoes a flip bifurcation when

$$
r=\frac{2}{\left(a_{1}+2 a_{2} x^{*}\right) x^{*}} .
$$

\section{Direction and Stability of the Neimark-Sacker Bifurcation}

In this section, we will study the direction and the stability of the Neimark-Sacker bifurcation in model (4). The method we used is based on the techniques developed by Yuri [19].

Without loss of generality, denote $\omega_{2 k-1}$ by $\omega^{*}$ and the critical value $r_{k}(k=1,2, \ldots,[(m-1) / 2]+1)$ by $r^{*}$ at which map (7) undergoes a Neimark-Sacker bifurcation at the origin.

For map (7), we obtain

$$
Y_{n+1}=A Y_{n}+\frac{1}{2} B\left(Y_{n}, Y_{n}\right)+\frac{1}{6} C\left(Y_{n}, Y_{n}, Y_{n}\right)+O\left(\left\|Y_{n}\right\|^{4}\right)
$$

where

$$
\begin{gathered}
B\left(Y_{n}, Y_{n}\right)=\left(B_{0}\left(Y_{n}, Y_{n}\right), 0, \ldots, 0\right)^{T}, \\
C\left(Y_{n}, Y_{n}, Y_{n}\right)=\left(C_{0}\left(Y_{n}, Y_{n}, Y_{n}\right), 0, \ldots, 0\right)^{T}, \\
B_{0}\left(Y_{n}, Y_{n}\right)=-2 r\left(a_{1}+2 a_{2} x^{*}\right) y_{n-m} y_{n} \\
+\left[\left(r\left(a_{1}+2 a_{2} x^{*}\right)\right)^{2}-2 r a_{2}\right] x^{*} y_{n-m}^{2}, \\
C_{0}\left(Y_{n}, Y_{n}, Y_{n}\right) \\
=3\left[r^{2}\left(a_{1}+2 a_{2} x^{*}\right)^{2}-2 r a_{2}\right] y_{n} y_{n-m}^{2} \\
+\left\{6 a_{2} r^{2}\left(a_{1}+2 a_{2} x^{*}\right)-\left[r\left(a_{1}+2 a_{2} x^{*}\right)\right]^{3}\right\} x^{*} y_{n-m}^{3}
\end{gathered}
$$


Let $q \in \mathbb{C}^{m+1}$ be a complex eigenvector corresponding to $e^{i \omega^{*}}$,

$$
A q=e^{i \omega^{*}} q, \quad A \bar{q}=e^{-i \omega^{*}} \bar{q} .
$$

We also introduce an adjoint eigenvector $q^{*} \in \mathbb{C}^{m+1}$ having the properties

$$
A^{T} q^{*}=e^{-i \omega^{*}} q^{*}, \quad A^{T} \overline{q^{*}}=e^{i \omega^{*}} \overline{q^{*}}
$$

and satisfying the normalization $\left\langle q^{*}, q\right\rangle=1$, where $\left\langle q^{*}, q\right\rangle=$ $\sum_{j=0}^{m} \overline{q_{j}^{*}} q_{j}$.

Lemma 6. Let $q=\left(q_{0}, q_{1}, \ldots, q_{m}\right)^{T}$ be the eigenvector of $A$ corresponding to eigenvalue $e^{i \omega^{*}}$ and let $q^{*}=\left(q_{0}^{*}, q_{1}^{*}, \ldots, q_{m}^{*}\right)^{T}$ be the eigenvector of $A^{T}$ corresponding to eigenvalue $e^{-i \omega^{*}}$. Then

$$
\begin{gathered}
q=\left(1, e^{-i \omega^{*}}, e^{-2 i \omega^{*}}, \ldots, e^{-m i \omega^{*}}\right)^{T}, \\
q^{*}=\left(\begin{array}{c}
q_{0}^{*} \\
-e^{i m \omega^{*}} r^{*}\left(a_{1}+2 a_{2} x^{*}\right) x^{*} q_{0}^{*} \\
-e^{i(m-1) \omega^{*}} r^{*}\left(a_{1}+2 a_{2} x^{*}\right) x^{*} q_{0}^{*} \\
\vdots \\
-e^{i \omega^{*}} r^{*}\left(a_{1}+2 a_{2} x^{*}\right) x^{*} q_{0}^{*}
\end{array}\right),
\end{gathered}
$$

where

$$
q_{0}^{*}=\frac{1}{1-e^{-i(m+1) \omega^{*}} r^{*}\left(a_{1}+2 a_{2} x^{*}\right) x^{*} m} .
$$

Proof. Let $q=\left(q_{0}, q_{1}, \ldots, q_{m}\right)^{T}$ be an eigenvector of $A$ corresponding to eigenvalue $e^{i \omega^{*}}$. Then

$$
\begin{gathered}
q_{0}-r^{*}\left(a_{1}+2 a_{2} x^{*}\right) x^{*} q_{m}=e^{i \omega^{*}} q_{0}, \\
q_{j}=e^{i \omega^{*}} q_{j+1}, \quad j=0,1,2, \ldots, m-1 .
\end{gathered}
$$

Similarly,

$$
q^{*}=\left(\begin{array}{c}
q_{0}^{*} \\
-e^{i m \omega^{*}} r^{*}\left(a_{1}+2 a_{2} x^{*}\right) x^{*} q_{0}^{*} \\
-e^{i(m-1) \omega^{*}} r^{*}\left(a_{1}+2 a_{2} x^{*}\right) x^{*} q_{0}^{*} \\
\vdots \\
-e^{i \omega^{*}} r^{*}\left(a_{1}+2 a_{2} x^{*}\right) x^{*} q_{0}^{*}
\end{array}\right) .
$$

For satisfying the normalization $\left\langle q^{*}, q\right\rangle=1$, we choose

$$
q_{0}^{*}=\frac{1}{1-e^{-i(m+1) \omega^{*}} r^{*}\left(a_{1}+2 a_{2} x^{*}\right) x^{*} m} .
$$

Let $T^{\mathcal{c}}$ denote a real eigenspace corresponding to $e^{ \pm i \omega^{*}}$, which is two-dimensional and is spanned by $\{\mathfrak{R}(q), \mathfrak{J}(q)\}$, and let $T^{\mathcal{S}}$ be a real eigenspace corresponding to all eigenvalues of $A^{T}$ other than $e^{ \pm i \omega^{*}}$ being $(m-1)$ dimensional.

For any $u \in \mathbb{R}^{m+1}$, we have its decomposition

$$
u=w q+\overline{w q}+v,
$$

where $w \in \mathbb{C}, w q+\overline{w q} \in T^{c}$, and $v \in T^{s}$. The complex variable $w$ can be viewed as a new coordinate on $T^{c}$, and

$$
\begin{gathered}
w=\left\langle q^{*}, u\right\rangle, \\
v=u-\left\langle q^{*}, u\right\rangle q-\left\langle\overline{q^{*}}, u\right\rangle \bar{q} .
\end{gathered}
$$

In this coordinate, the map $F$ at $r=r^{*}$ has the form

$$
\begin{gathered}
w \longmapsto e^{i \omega^{*}} w+\left\langle q^{*}, F(w q+\overline{w q}+v)\right\rangle, \\
v \longmapsto A v+F(w q+\overline{w q}+v)-\left\langle q^{*}, F(w q+\overline{w q}+v)\right\rangle q \\
-\left\langle\overline{q^{*}}, F(w q+\overline{w q}+v)\right\rangle \bar{q} .
\end{gathered}
$$

Using Taylor expansions, we obtain

$$
\begin{gathered}
w \longmapsto e^{i \omega^{*}} w+\frac{1}{2} g_{20} w^{2}+g_{11} w \bar{w}+\frac{1}{2} g_{02} \bar{w}^{2} \\
+\frac{1}{2} G_{21} w^{2} \bar{w}+\left\langle G_{10}, v\right\rangle w+\left\langle G_{01}, v\right\rangle \bar{w}, \\
v \longmapsto A v+\frac{1}{2} H_{20} w^{2}+H_{11} w \bar{w}+\frac{1}{2} H_{02} \bar{w}^{2}+O\left(|w|^{3}\right),
\end{gathered}
$$

where $g_{i j} \in \mathbb{C}, G_{10}, G_{01} \in \mathbb{C}^{m+1}$, and

$$
\begin{gathered}
g_{20}=\left\langle q^{*}, B(q, q)\right\rangle, \quad g_{11}=\left\langle q^{*}, B(q, \bar{q})\right\rangle, \\
g_{02}=\left\langle q^{*}, B(\bar{q}, \bar{q})\right\rangle, \\
G_{21}=\left\langle q^{*}, C(q, q, \bar{q})\right\rangle, \quad\left\langle G_{10}, v\right\rangle=\left\langle q^{*}, B(q, v)\right\rangle, \\
\left\langle G_{01}, v\right\rangle=\left\langle q^{*}, B(\bar{q}, v)\right\rangle, \\
H_{20}=B(q, q)-\left\langle q^{*}, B(q, q)\right\rangle q-\left\langle\overline{q^{*}, B}(q, q)\right\rangle \bar{q}, \\
H_{11}=B(q, \bar{q})-\left\langle q^{*}, B(q, \bar{q})\right\rangle q-\left\langle\overline{q^{*}}, B(q, \bar{q})\right\rangle \bar{q}, \\
H_{02}=B(\bar{q}, \bar{q})-\left\langle q^{*}, B(q, \bar{q})\right\rangle q-\left\langle\overline{q^{*}}, B(\bar{q}, \bar{q})\right\rangle \bar{q} .
\end{gathered}
$$

Now, we seek the center manifold which has the representation

$$
v=V(w, \bar{w})=\frac{1}{2} z_{20} w^{2}+z_{11} w \bar{w}+\frac{1}{2} z_{02} \bar{w}^{2}+O\left(|w|^{3}\right),
$$

where $\left\langle q^{*}, z_{i j}\right\rangle=0$. Substituting (40) into (38), we have

$$
\begin{gathered}
z_{20}=\left(e^{2 i \omega^{*}} I-A\right)^{-1} H_{20}, \quad z_{11}=(I-A)^{-1} H_{11}, \\
z_{02}=\left(e^{-2 i \omega^{*}} I-A\right)^{-1} H_{02} .
\end{gathered}
$$




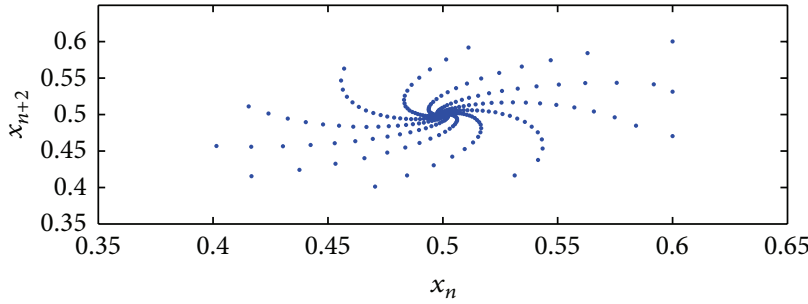

(a)

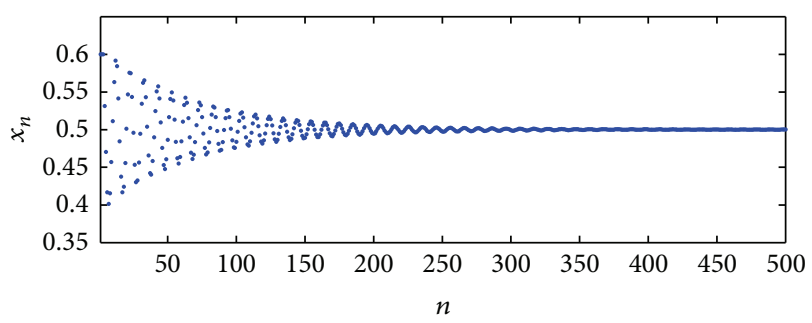

(b)

FIGURE 1: The positive equilibrium is asymptotically stable, where $a_{1}=1.6, a_{2}=0.8, m=2$, and $r=0.49<r_{1}=0.515$.

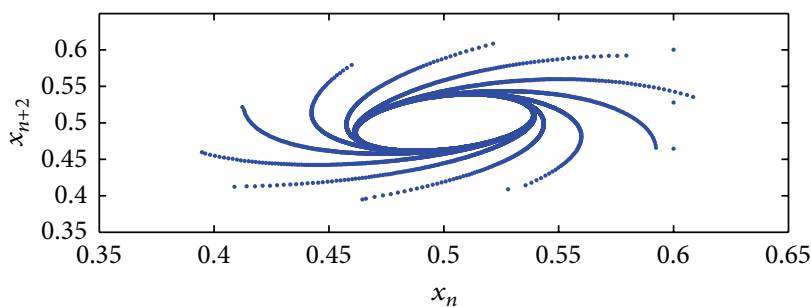

(a)

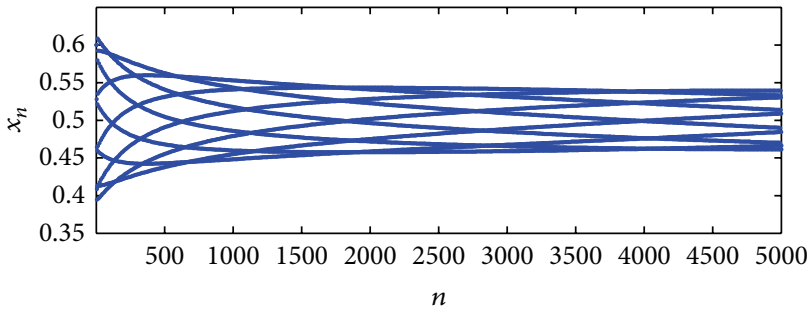

(b)

FIGURE 2: A stable invariant closed circle bifurcating from $x^{*}$, where $a_{1}=1.6, a_{2}=0.8, m=2$, and $r=0.5156>r_{1}=0.515$.

When restricting (38) to the center manifold, up to cubic term, then we have

$$
\begin{gathered}
g_{20}=\left\langle q^{*}, B(q, q)\right\rangle, \quad g_{11}=\left\langle q^{*}, B(q, \bar{q})\right\rangle, \\
g_{02}=\left\langle q^{*}, B(\bar{q}, \bar{q})\right\rangle, \\
g_{21}=\left\langle q^{*}, B\left(\bar{q}, z_{20}\right)\right\rangle+2\left\langle q^{*}, B\left(q, z_{11}\right)\right\rangle+\left\langle q^{*}, C(q, q, \bar{q})\right\rangle \\
=\left\langle q^{*}, C(q, q, \bar{q})\right\rangle-2\left\langle q^{*}, B\left(q,\left(I-A^{-1}\right) B(q, \bar{q})\right)\right\rangle \\
+\left\langle q^{*}, B\left(\bar{q},\left(e^{2 i \omega^{*}} I-A\right)^{-1} B(q, q)\right)\right\rangle \\
+\frac{e^{-i \omega^{*}}\left(1-2 e^{2 i \omega^{*}}\right)}{1-e^{-i \omega^{*}}}\left\langle q^{*}, B(q, q)\right\rangle\left\langle q^{*}, B(q, \bar{q})\right\rangle \\
-\frac{2}{1-e^{-i \omega^{*}}}\left|\left\langle q^{*}, B(\bar{q}, \bar{q})\right\rangle\right|^{2} \\
-\frac{e^{i \omega^{*}}}{e^{3 i \omega^{*}}-1}\left|\left\langle q^{*}, B(\bar{q}, \bar{q})\right\rangle\right|^{2} .
\end{gathered}
$$

Define

$$
c(r)=\frac{g_{20} g_{11}(\bar{w}-3+2 w)}{2\left(w^{2}-w\right)(\bar{w}-1)}+\frac{\left|g_{11}\right|^{2}}{1-\bar{w}}+\frac{\left|g_{02}\right|^{2}}{2\left(w^{2}-\bar{w}\right)}+\frac{g_{21}}{2} .
$$

Substituting $w=e^{-i \omega^{*}}$ into (43), we can obtain $c\left(r^{*}\right)$.

From the above argument, we have the following result.
Theorem 7. The direction and stability of Neimark-Sacker bifurcation of model (4) can be determined by the sign of $\mathfrak{R}\left[e^{-i \omega^{*}} c\left(r^{*}\right)\right]$; that is, the Neimark-Sacker bifurcation of model (4) at $r=r_{j}$ is supercritical (resp., subcritical) and unique closed invariant curve bifurcation from $x^{*}$ is asymptotically stable (resp., unstable) when $\mathfrak{R}\left[e^{-i \omega^{*}} c\left(r^{*}\right)\right]<0$ (resp., $\mathfrak{R}\left[e^{-i \omega^{*}} c\left(r^{*}\right)\right]>0$ ).

\section{Numerical Simulations}

In this section we will give an example to illustrate the analytic results. Here we take model (4) with $a_{1}=1.6, a_{2}=0.8, m=2$, and $m=5$, respectively. From $a_{1}=1.6, a_{2}=0.8$, and $m=2$, we have $x^{*}=0.5$ and $r_{1}=0.515$. By the simple calculation, we obtain $\mathfrak{R}\left[e^{-i \omega^{*}} c\left(r^{*}\right)\right]=-0.1452$. Similarly, when $m=5$, we have $x^{*}=0.5, r_{1}=0.2372$, and $\mathfrak{R}\left[e^{-i \omega^{*}} c\left(r^{*}\right)\right]=-0.0948$.

From Theorem 5, we conclude that, with $a_{1}=1.6, a_{2}=$ 0.8 , and $m=2$, the positive equilibrium $x^{*}=0.5$ of model (4) is asymptotically stable when $r<r_{1}=0.515$, and model (4) undergoes the Neimark-Sacker bifurcation at $x^{*}$ when $r=r_{1}=0.515$. Furthermore, from Theorem 7 and $\mathfrak{R}\left[e^{-i \omega^{*}} c\left(r^{*}\right)\right]=-0.1452<0$ the Neimark-Sacker bifurcation of model (4) at $r=r_{1}$ is supercritical and unique closed invariant curve bifurcating from $x^{*}$ is asymptotically stable. These are shown by Figures 1 and 2 .

Similarly, with $a_{1}=1.6, a_{2}=0.8$, and $m=5$, the positive equilibrium $x^{*}=0.5$ of model (4) is asymptotically stable when $r<r_{1}=0.2372$, and, from Theorem 7 and $\mathfrak{R}\left[e^{-i \omega^{*}} c\left(r^{*}\right)\right]=-0.0948<0$, the Neimark-Sacker bifurcation of model (4) at $r=r_{1}$ is supercritical and unique 


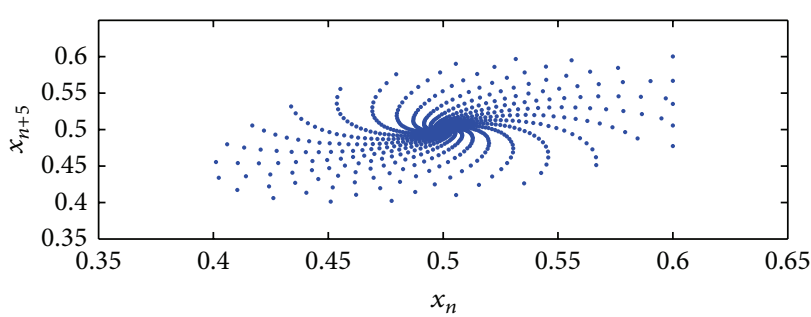

(a)

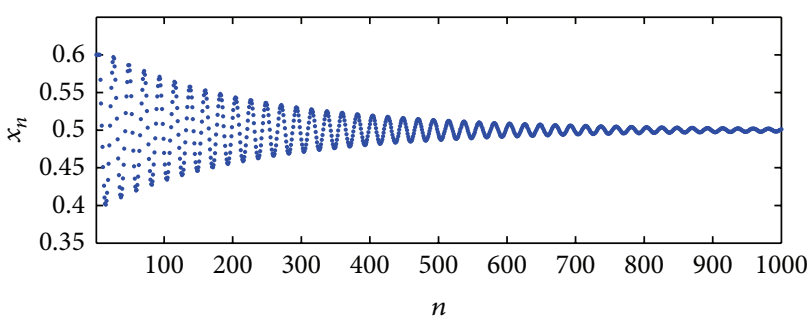

(b)

FIGURE 3: The positive equilibrium is asymptotically stable, where $a_{1}=1.6, a_{2}=0.8, m=5$, and $r=0.23<r_{1}=0.2372$.

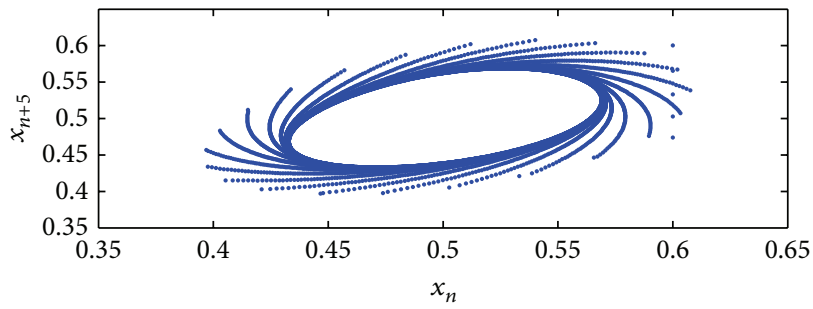

(a)

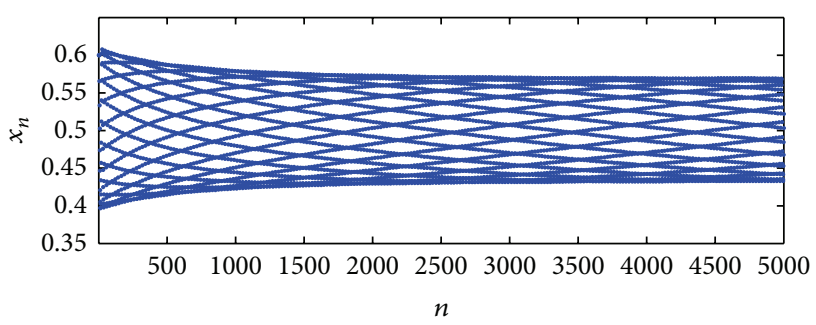

(b)

FIGURE 4: A stable invariant closed circle bifurcating from $x^{*}$, where $a_{1}=1.6, a_{2}=0.8, m=5$, and $r=0.238>r_{1}=0.2372$.

closed invariant curve bifurcating from $x^{*}$ is asymptotically stable. These are shown by Figures 3 and 4 .

\section{Conclusion and Discussion}

There has been a large body of work discussing the stability and bifurcation in logistic model, but most of them dealt with only the continuous logistic models, or only the discrete logistic model without delays. In this paper, we discuss the dynamical behaviors of nonlinear discrete logistic model with delay. The characteristic equation (11) is a polynomial equation involving high order terms, which make it difficult to find all parameters such that the characteristic roots have modulus 1 or less. By analyzing the characteristic equation, some sufficient and necessary conditions are derived to ensure that all the characteristic roots have modulus less than 1. Moreover, we obtain that when the parameter $r$ varies, the positive equilibrium of the model (4) exchanges its stability and Neimark-Sacker bifurcation occurs. Furthermore, the direction and stability of closed invariant curve from the Neimark-Sacker bifurcation of the model (4) are determined. Numerical simulations also show the occurrence of the stable bifurcate periodic solutions when $r$ passes the critical $r^{*}$.

\section{Conflict of Interests}

The authors declare that there is no conflict of interests regarding the publication of this paper.

\section{Acknowledgments}

The authors are very grateful to the Associate Editor, Professor Zhan Zhou, and the anonymous reviewers for their valuable time and professional comments. This paper is jointly supported by the National Natural Science Foundation of China under Grant nos. 11072059 and 61272530, the Specialized Research Fund for the Doctoral Program of Higher Education under Grant no. 20110092110017, the Natural Science Foundation of Jiangsu Province of China under Grant no. BK2012741, and the Programs of Educational Commission of Anhui Province of China under Grant nos. KJ2011A197 and KJ2013Z186. This work is also funded by the Deanship of Scientific Research, King Abdulaziz University (KAU), under Grant no. 3-130/1434/HiCi. Therefore, the authors acknowledge technical and financial support of KAU.

\section{References}

[1] A. K. Laird, "Dynamics of tumour growth," British Journal of Cancer, vol. 13, pp. 490-502, 1964.

[2] A. K. Laird, "Dynamics of tumour growth: comparison of growth rates and extrapolation of growth curve to one cell," British Journal of Cancer, vol. 19, pp. 278-291, 1965.

[3] J. C. Panetta, "A logistic model of periodic chemotherapy," Applied Mathematics Letters, vol. 8, no. 4, pp. 83-86, 1995.

[4] J. C. Panetta, "A logistic model of periodic chemotherapy with drug resistance," Applied Mathematics Letters, vol. 10, no. 1, pp. 123-127, 1997.

[5] M. Bodnar and U. Forys, “Three types of simple DDE's describing tumour growth," Journal of Biological Systems, vol. 15, no. 4, pp. 1-19, 2007.

[6] H. V. Jaina and H. M. Byrne, "Qualitative analysis of an integrodifferential equation model of periodic chemotherapy," Applied Mathematics Letters, vol. 25, no. 12, pp. 2132-2136, 2012.

[7] K. Gopalsamy, Stability and Oscillations in Delay Differential Equtions of Population Dynamics, Kluwer Academic, Boston, Mass, USA, 1992. 
[8] F. D. Chen, X. D. Xie, and X. X. Chen, "Permanence and global attractivity of a delayed periodic logistic equation," Applied Mathematics and Computation, vol. 177, no. 1, pp. 118-127, 2006.

[9] M. Bodnar, U. Forys et al., "Logistic type equations with discrete delay and quasi-periodic suppression rate," Applied Mathematics Letters, vol. 26, no. 6, pp. 607-611, 2013.

[10] S. Mohamad and K. Gopalsamy, "Extreme stability and almost periodicity in a discrete logistic equation," Tohoku Mathematical Journal, vol. 52, no. 1, pp. 107-125, 2000.

[11] Y. Muroya, "Global attractivity for discrete models of nonautonomous logistic equations," Computers and Mathematics with Applications, vol. 53, no. 7, pp. 1059-1073, 2007.

[12] Z. Li and F. D. Chen, "Almost periodic solutions of a discrete almost periodic logistic equation," Mathematical and Computer Modelling, vol. 50, no. 1-2, pp. 254-259, 2009.

[13] Z. Zhou and X. Zou, "Stable periodic solutions in a discrete periodic logistic equation," Applied Mathematics Letters, vol. 16, no. 2, pp. 165-171, 2003.

[14] H. R. Sun and W. T. Li, "Qualitative analysis of a discrete logistic equation with several delays," Applied Mathematics and Computation, vol. 147, no. 2, pp. 515-525, 2004.

[15] Y. M. Chen and Z. Zhou, "Global attractivity and oscillation in a nonlinear periodic delay difference equation," Computers and Mathematics with Applications, vol. 45, no. 6-9, pp. 943-950, 2003.

[16] Q. Liu, R. Xu, and Z. Wang, "Stability and bifurcation of a class of discrete-time Cohen-Grossberg neural networks with delays," Discrete Dynamics in Nature and Society, vol. 2011, Article ID 403873, 14 pages, 2011.

[17] W. Han and M. Liu, "Stability and bifurcation analysis for a discrete-time model of Lotka-Volterra type with delay," Applied Mathematics and Computation, vol. 217, no. 12, pp. 5449-5457, 2011.

[18] W. He and J. Cao, "Stability and bifurcation of a class of discretetime neural networks," Applied Mathematical Modelling, vol. 31, no. 10, pp. 2111-2122, 2007.

[19] A. K. Yuri, Elements of Applied Bifurcation Theory, Springer, New York, NY, USA, 1995.

[20] S. J. Guo, X. H. Tang, and L. H. Huang, "Stability and bifurcation in a discrete system of two neurons with delays," Nonlinear Analysis: Real World Applications, vol. 9, no. 4, pp. 1323-1335, 2008. 


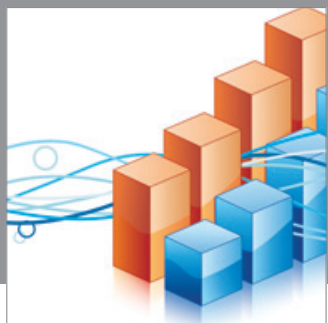

Advances in

Operations Research

mansans

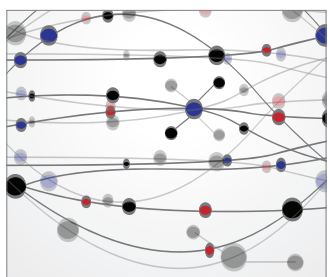

The Scientific World Journal
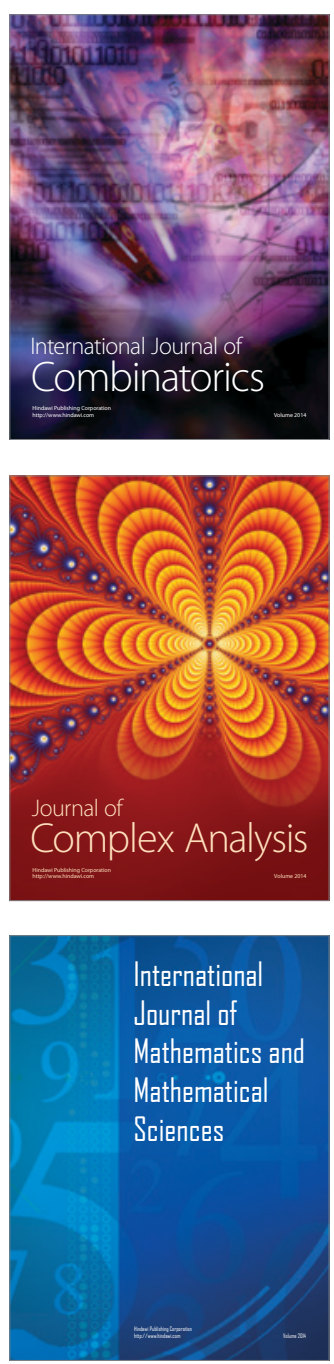
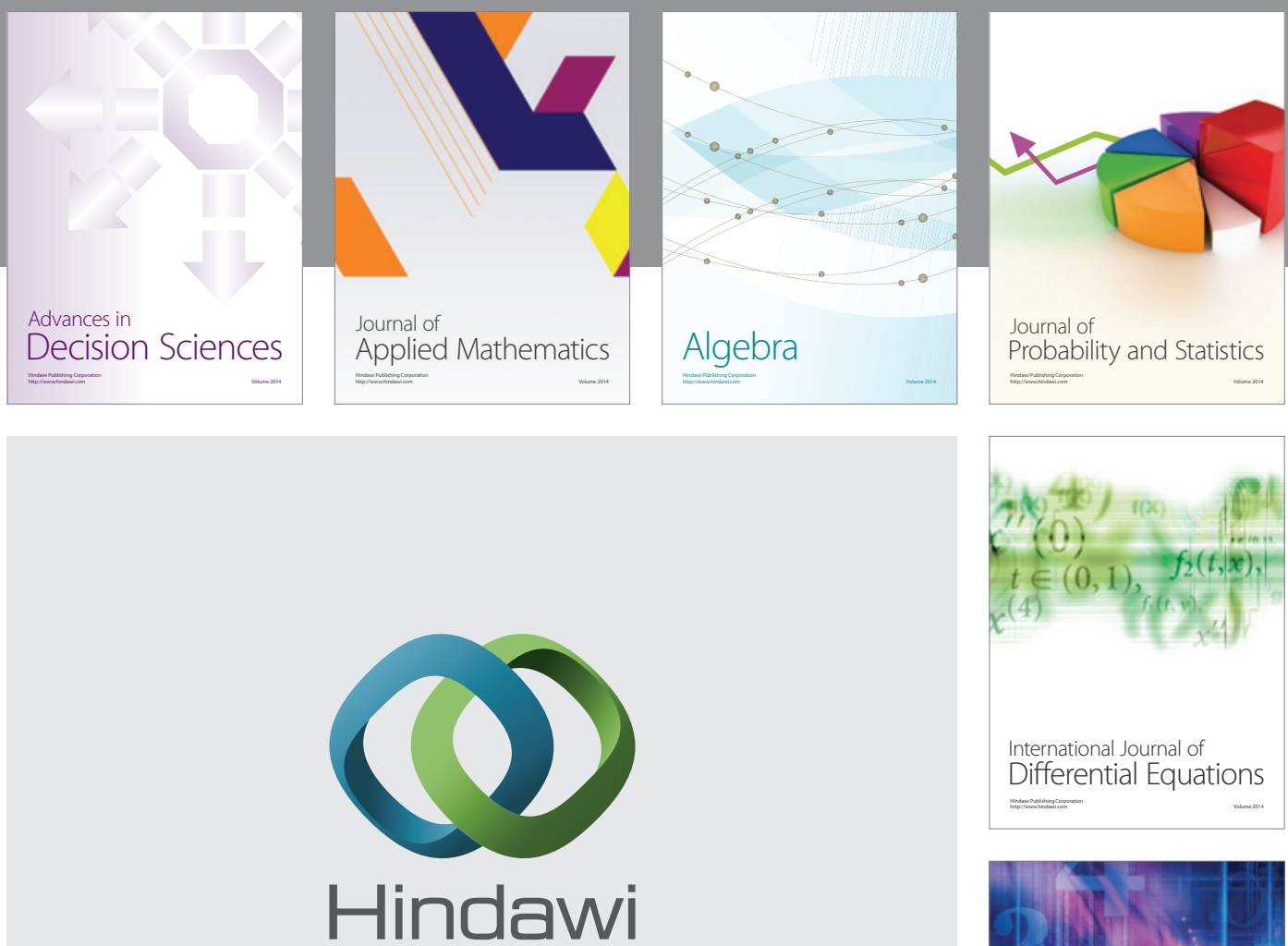

Submit your manuscripts at http://www.hindawi.com
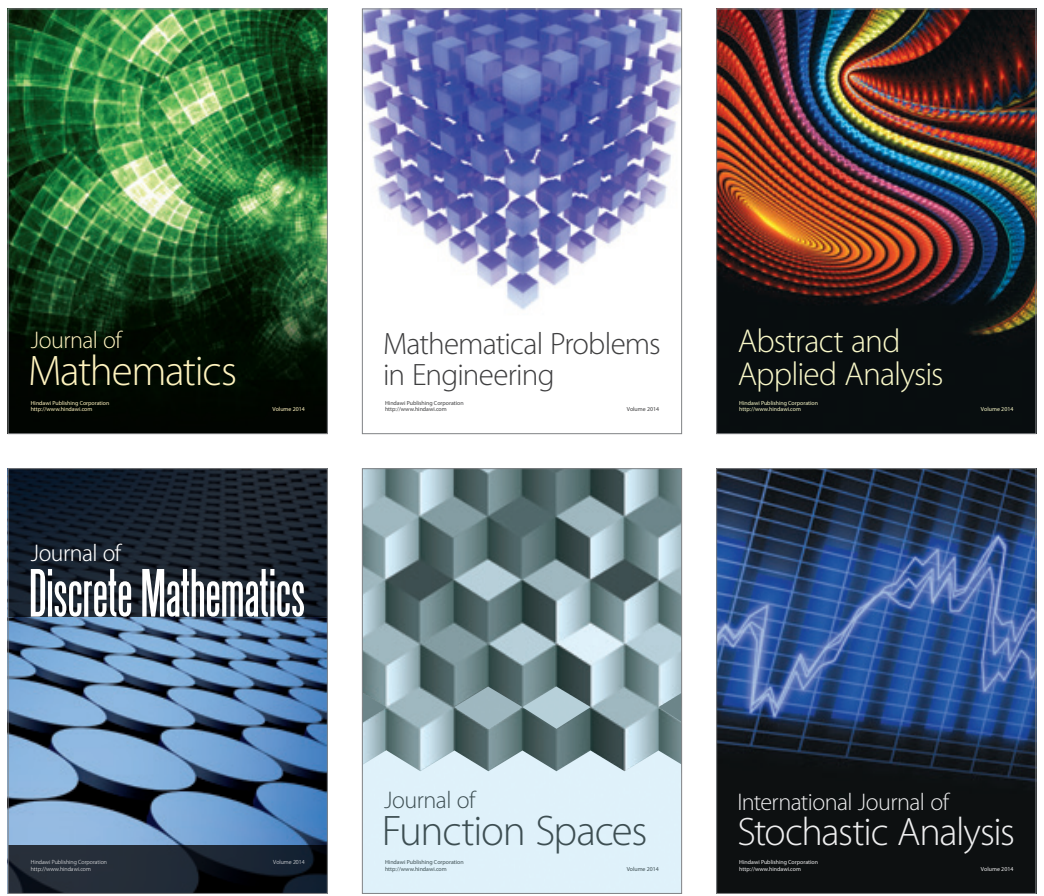

Journal of

Function Spaces

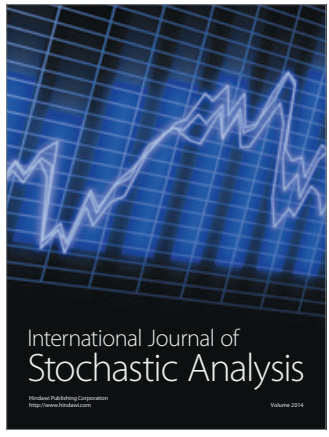

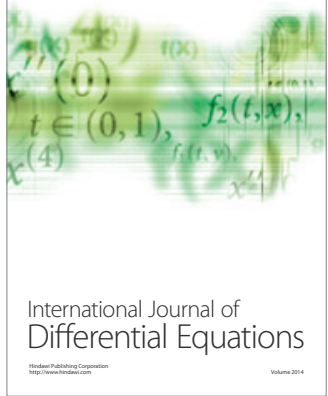
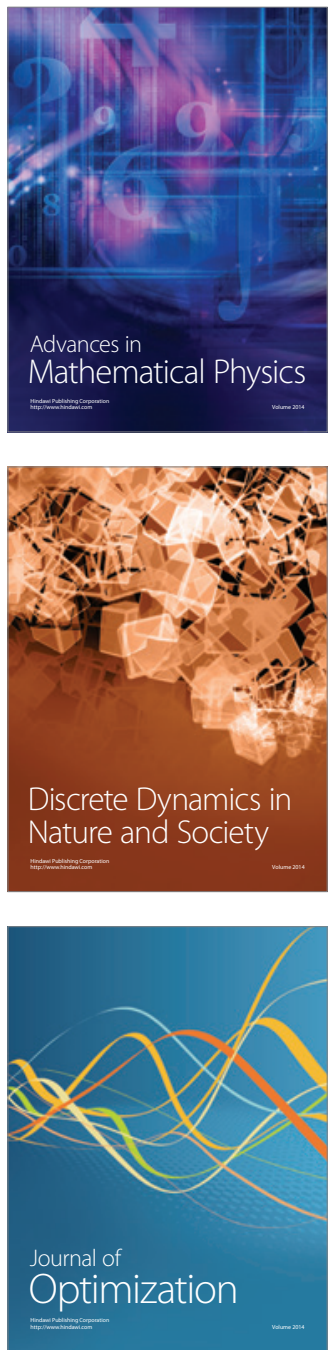